\title{
Facial Diplegia as a Rare Late Neurologic Manifestation of SARS-CoV-2 Infection
}

\author{
Casey Judge ${ }^{\mathrm{a}}$, Negar Moheb ${ }^{\mathrm{a}}$, Ramiro Castro Apolo ${ }^{\mathrm{a}}$, Joy L. Dupont ${ }^{\mathrm{a}}$, \\ Michelle L. Gessner ${ }^{\mathrm{a}}$, Hussam A. Yacoub ${ }^{\mathrm{a}, \mathrm{b}}$
}

\begin{abstract}
Multiple recent publications have reported numerous neurologic complications of severe acute respiratory syndrome coronavirus 2 (SARS$\mathrm{CoV}-2$ ) infection. Among these is Guillain-Barre syndrome and its variants, including facial diplegia. In this case we present a patient with facial diplegia following a confirmed SARS-CoV-2 infection. The patient initially presented with respiratory symptoms and subsequently developed bilateral facial weakness approximately 3 weeks later prompting an emergency department (ED) visit. Extensive laboratory and imaging workup was negative for other etiologies. Cerebrospinal fluid (CSF) analysis was notable only for mild elevation in white blood cells and protein. Patients with acute neurologic symptoms should be evaluated carefully regarding recent infections or possible exposures to help identify and minimize late complications of this novel virus.
\end{abstract}

Keywords: COVID-19; Viral infections; Facial diplegia

\section{Introduction}

Neurological manifestations of the severe acute respiratory syndrome coronavirus 2 (SARS-CoV-2) are rare. Among these are encephalitis, anosmia and ageusia, acute cerebrovascular disease including ischemic stroke, and Guillain-Barre syndrome (GBS) and its variants. Facial diplegia (FD) is a rarely reported late neurologic complication of this novel virus. We report a case of a patient who presented with FD approximately 3 weeks following confirmed SARS-CoV-2 infection.

\section{Case Report}

A 64-year-old man initially presented to an outpatient clin-

Manuscript submitted June 15, 2020, accepted June 19, 2020

Published online December 9, 2020

aDepartment of Neurology, Lehigh Valley Health Network, Allentown, PA, USA

${ }^{b}$ Corresponding Author: Hussam A. Yacoub, Department of Neurology, Lehigh Valley Health Network, 1250 South Cedar Crest Blvd., Suite 405, Allentown, PA 18103, USA. Email: hussam_a.yacoub@1vhn.org

doi: https://doi.org/10.14740/jnr606 ic with complaints of cough, fever, and chills for 1 week. A SARS-CoV-2 polymerase chain reaction (PCR) test was positive, and the patient was advised to self-quarantine. He recovered completely from the respiratory symptoms. Approximately 3 weeks following the onset of respiratory symptoms, he presented to the emergency department with complaints of progressive bilateral facial weakness, dysarthria, and subjective facial paresthesia for the previous 2 days. On examination, he was observed to have bilateral facial nerve palsy in a peripheral distribution, more pronounced on the right. The remaining cranial nerves appeared normal. Motor, sensory, coordination, and gait examination was unremarkable.

Repeat SARS-CoV-2 PCR was negative. Magnetic resonance imaging of the brain with and without gadolinium was unremarkable for any acute pathology. Cerebrospinal fluid (CSF) analysis revealed lymphocytic pleocytosis of 10 white blood cells (WBCs) $/ \mathrm{mm}^{3}$ (100\% lymphocytes), mild elevation of protein level at $53 \mathrm{mg} / \mathrm{dL}$, and normal glucose at $66 \mathrm{mg} / \mathrm{dL}$. Lyme serologies revealed elevation in immunoglobulin $\mathrm{M}(\operatorname{IgM})$ antibody index at 1.57 (reference range $<0.91$ ) but confirmatory Western blot was negative. Lyme CSF antibody index was negative. Additional infectious workup including cytomegalovirus (CMV) and human T-lymphotropic virus was negative. Remaining neurologic workup including acetylcholine receptor antibodies (binding, blocking, modulating), muscle-specific tyrosine kinase (MuSK) antibody, ganglioside antibodies (including antiGQ1b), serum angiotensin converting enzyme level was unremarkable. Patient was ultimately discharged in a stable condition with gradual improvement of the facial weakness and paresthesia.

\section{Discussion}

Multiple neurologic complications of the SARS-CoV-2 infection have been identified. Possible mechanisms of neurologic injury include direct neurotropism, which has been postulated to be the mechanism underlying anosmia/ageusia and encephalitis, systemic inflammatory responses resulting in hypoxic or toxic-metabolic nervous system injury, or a delayed onset autoimmune-mediated mechanism. While GBS is known to be a parainfectious complication of multiple viruses including influenza, Zika virus, and CMV, to our knowledge, less than 15 reports of delayed onset SARS- 
CoV-2 related Guillain-Barre-like complications have been identified [1]. The majority of cases describe acute onset of para- or tetraplegia in the setting of known SARS-CoV-2 infection [2-4]. In reported cases, a 2 - 3 week latent period from time of infection to neurologic symptom onset was observed, which may suggest an autoimmune etiology [3, 4]. In contrast to our case, CSF analysis did not reveal pleocytosis or increased protein level [2-4]. Only one other case of FD related to SARS-CoV-2 has been reported [5], with mild elevation of CSF protein level at $44 \mathrm{mg} / \mathrm{dL}$. Our case report is in concordance with previous literature reporting an association of FD variant GBS with negative ganglioside antibodies [6].

\section{Conclusions}

A growing amount of evidence suggests that SARS-CoV-2 has a multitude of neurologic manifestations. To our knowledge, our case is only the second reported case of FD following a SARS-CoV-2 infection. Patients presenting with symptoms suggestive of acute neurologic disorders should be evaluated carefully regarding recent infectious symptoms or possible exposures to help identify and minimize late complications of this novel virus.

\section{Acknowledgments}

None to declare.

\section{Financial Disclosure}

No funding was received. None of the authors have disclosures relevant to this manuscript.

\section{Conflict of Interest}

None to declare.

\section{Informed Consent}

Not applicable.

\section{Author Contributions}

All authors participated in data collection, data interpretation and editing the manuscript.

\section{Data Availability}

The authors declare that data supporting the findings of this study are available within the article.

\section{References}

1. Gutierrez-Ortiz C, Mendez-Guerrero A, Rodrigo-Rey S, San Pedro-Murillo E, Bermejo-Guerrero L, Gordo-Manas R, de Aragon-Gomez F, et al. Miller Fisher syndrome and polyneuritis cranialis in COVID-19. Neurology. 2020;95(5):e601-e605.

2. Zhao H, Shen D, Zhou H, Liu J, Chen S. Guillain-Barre syndrome associated with SARS-CoV-2 infection: causality or coincidence? Lancet Neurol. 2020;19(5):383-384.

3. Sedaghat Z, Karimi N. Guillain Barre syndrome associated with COVID-19 infection: A case report. J Clin Neurosci. 2020;76:233-235.

4. Scheidl E, Canseco DD, Hadji-Naumov A, Bereznai B. Guillain-Barre syndrome during SARS-CoV-2 pandemic: A case report and review of recent literature. J Peripher Nerv Syst. 2020;25(2):204-207.

5. Juliao Caamano DS, Alonso Beato R. Facial diplegia, a possible atypical variant of Guillain-Barre Syndrome as a rare neurological complication of SARS-CoV-2. J Clin Neurosci. 2020;77:230-232.

6. Kim JK, Oh SY, Sohn EH, Hong YH, Jun SM, Bae JS. When is facial diplegia regarded as a variant of GuillainBarre syndrome? J Peripher Nerv Syst. 2015;20(1):32-36. 